\title{
Abnormal lipoprotein metabolism and reversible female infertility in HDL receptor (SR-BI)-deficient mice
}

\author{
Helena E. Miettinen, Helen Rayburn, and Monty Krieger \\ Department of Biology, Massachusetts Institute of Technology, Cambridge, Massachusetts, USA \\ Address correspondence to: Monty Krieger, Room 68-483, Biology Department, Massachusetts Institute of Technology, \\ Cambridge, Massachusetts 02139, USA. Phone: (617) 253-6793; Fax: (617) 258-5851; E-mail: krieger@mit.edu. \\ Helena E. Miettinen's present address is: Department of Medicine, University of Helsinki, Helsinki, Finland. \\ Helen Rayburn's present address is: Department of Anatomy, University of California, San Francisco, San Francisco, California, USA. \\ Received for publication May 17, 2001, and accepted in revised form October 8, 2001.
}

\begin{abstract}
Mammalian female fertility depends on complex interactions between the ovary and the extraovarian environment (e.g., the hypothalamic-hypophyseal ovarian axis). The role of plasma lipoproteins in fertility was examined using HDL-receptor SR-BI knockout (KO) mice. SR-BI KO females have abnormal HDLs, ovulate dysfunctional oocytes, and are infertile. Fertility was restored when the structure and/or quantity of abnormal HDL was altered by inactivating the apoAI gene or administering the cholesterol-lowering drug probucol. This suggests that abnormal lipoprotein metabolism can cause murine infertility - implying a functional hepatic-ovarian axis - and may contribute to some forms of human female infertility.
\end{abstract}

J. Clin. Invest. 108:1717-1722 (2001). DOI:10.1172/JCI200113288.

\section{Introduction}

Human female infertility, which in many cases is of unknown etiology, is a significant medical problem $(1,2)$. The influence of abnormal lipoprotein metabolism on female infertility has not been thoroughly explored, despite observations suggesting a potential role for plasma lipoproteins, especially HDL (3-5). Lipoproteins transport between tissues a number of lipids (e.g., cholesterol, steroid hormones, and vitamin E) that either directly, or indirectly through their metabolic products, play key roles in fertility $(6,7)$. In many species, including humans, the only lipoprotein detected in substantial amounts in the follicular fluid surrounding the developing oocyte in the ovary is HDL (8-10). HDL may deliver critical lipid nutrients to either the follicular cumulus cells or the oocytes for membrane synthesis, local steroid hormone production, or other processes essential for normal oocyte maturation. It might also have a role in cholesterol efflux (11-18) from the oocyte/cumulus cells, thus participating in the maintenance of cellular cholesterol balance. Therefore, abnormalities in HDL metabolism affecting its structure, abundance, or function might compromise female fertility.

Mice with homozygous null mutations in the gene for the HDL receptor SR-BI (SR-BI KO) provide an opportunity to explore the potential role of abnormal lipoprotein metabolism in infertility (18-20). Female, but not male, SR-BI KO mice are infertile, even though they exhibit normal estrus cycles, ovulation, and progesterone levels during pseudopregnancy (20). This infertility is due, at least in part, to the ovulation of dysfunctional oocytes (20). Because SR-BI is normally expressed in the uterus in the decidual cells (21) that nourish the implanted embryo and its expression in mammary glands is stimulated in rodents by pregnancy (22), it is possible that fertility and nursing might in part depend on SR-BI expression at extraovarian sites. Here we used genetic, surgical, and pharmacologic methods to study the infertility of SR-BI $\mathrm{KO}$ mice and report that lipoproteins can play a critical role in murine fertility.

\section{Methods}

Lipoprotein analysis. Plasma, collected from Avertin anesthetized mice, was size fractionated by FPLC (19), and the total cholesterol in each fraction or in unfractionated plasma was determined as described previously (19) or by using a commercial kit (Wako Chemical USA Inc., Richmond, Virginia, USA). For apoA-I KO mice, $500 \mu \mathrm{l}$ of pooled plasma was used for FPLC analyses.

Animals. Mice were housed and fed a normal chow diet (19) or chow (Teklad 7001) supplemented with $0.5 \%$ (wt/wt) 4,4'-(isopropylidene-dithio)-bis-(2,6-di-tertbutylphenol (probucol; Sigma Chemical Co., St. Louis, Missouri, USA). After ovary transplant surgery, the drinking water for RAG-2-deficient mice was supplemented with the antibiotics sulfamethoxazole $(1.0 \mathrm{mg} / \mathrm{ml})$ and trimethoprim $(0.2 \mathrm{mg} / \mathrm{ml})$ (Sulfatrim pediatrics; Alpharma U.S. Pharmaceuticals Division, Baltimore, Maryland, USA) to prevent postoperative infections.

Mouse strains (genetic backgrounds) were: wild-type and SR-BI KO (both 1:1 mixed C57BL/6 $\times 129$ backgrounds; ref. 19), apoA-I KO (23) (C57BL/6; The Jackson Laboratory, Bar Harbor, Maine, USA), and RAG-2 $\mathrm{KO}$ (mixed C57BL/ $6 \times 129 \times \mathrm{BALB} / \mathrm{c}$ background; gift from Jianzhu Chen, Massachusetts Institute of Technology). Double SR-BI/apoA-I KO and SR-BI/RAG-2 $\mathrm{KO}$ mice were produced by (a) mating SR-BI KO males with apoA-I KO or RAG-2-KO females, (b) transferring 


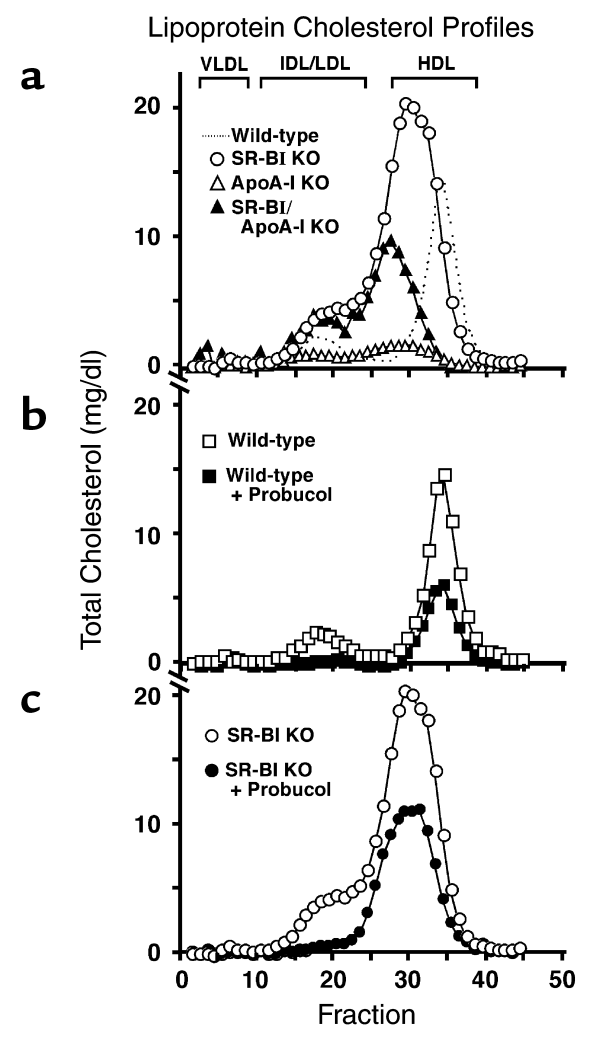

the resulting embryos into Swiss Webster recipients, and (c) intercrossing the double heterozygous offspring. Colonies were maintained by crossing double$\mathrm{KO}$ males with females heterozygous for the SR-BI null mutation and homozygous for the apoA-I or RAG-2 mutation to optimize the low yield of SR-BI homozygotes (19). It has been possible to obtain only a few homozygous null SR-BI KO mice on an essentially pure C57BL/ 6 background (backcrossed nine times to pure C57BL/6 mice). Experiments with these $(n=3$; 4month matings, no litters) and additional mixed background SR-BI KO mice ( $n=19$; not shown) confirmed the previously reported (20) extremely low fertility of female SR-BI KO mice. PCR was used for genotyping the various mutant mouse lines. The primers for analyses were as follows: for SR-BI, the primers were as previously described (19); for apoA-I, the following set of four primers were used: $5^{\prime}$-cttgggtggagaggctattc- $3^{\prime}$, $5^{\prime}$-aggtgagatgacaggagatc- $3^{\prime}, 5^{\prime}$-catctcgcacctttagccat- $3^{\prime}$, and $5^{\prime}$-tctctgtgcccaggaaggta- $3^{\prime}$ (sequences and protocols originally from the Jackson Laboratories), or we used primers $5^{\prime}$-acccagactgtcggagagct- $3^{\prime}$ and $5^{\prime}$-catcttgctgccatacgtgc- $3^{\prime}$; and for RAG-2, the primers were $5^{\prime}$-agcctgcttattgtctcctg- $3^{\prime}$ and $5^{\prime}$-ggcaccggacaggtcggtcttgac-3' (kindly provided by Charles Whitehurst, Massachusetts Institute of Technology). All animal studies were approved by the Massachusetts Institute of Technology Committee on Animal Care.

To test the fertility of genetically, surgically, or pharmacologically manipulated female mice, virgin females were housed continuously with wild-type males, and numbers of litters and pups were counted during the

\section{Figure 1}

Effects of genetic disruption of the apoA-I gene (a) or probucol treatment ( $\mathbf{b}$ and $\mathbf{c}$ ) on plasma lipoprotein profiles of wild-type and SR-BI KO mice. Plasma lipoproteins were separated by size-exclusion chromatography (Superose 6-FPLC), and total cholesterol was measured for each fraction (expressed as milligrams per deciliter of plasma). Approximate elution positions of VLDL, IDL/LDL, and HDL are indicated as described previously (19). Lipoprotein-cholesterol profiles of (a) wild-type (dashed line, pooled plasma from four mice); SR-BI KO mice (open circles, pooled plasma from seven mice); apoA-I KO mice (open triangles, pooled plasma from three mice); and SR-BI/apoA-I double-KO mice (filled triangles; average of three chromatograms from three mice); (b) wild-type mice fed first with normal chow (open squares, pooled plasma from four mice, same as dashes in a); then with $0.5 \%$ probucol-enriched chow for 11 days (filled squares, pooled plasma from four mice); and (c) SR-BI KO mice fed first with normal chow (open circles, pooled plasma from seven mice, same as in a); and then with $0.5 \%$ probucol-enriched chow for 25 days (filled circles, pooled plasma from seven mice). The average plasma total cholesterol levels ( \pm SEM) were: wild-type, $103.4 \pm 3.8 \mathrm{mg} / \mathrm{dl}(n=7)$; SR-BI KO, $211.5 \pm 6.2$ $\mathrm{mg} / \mathrm{dl}(n=13)$; apoA-I KO, $25 \pm 1.2 \mathrm{mg} / \mathrm{dl}(n=3)$; SR-BI/apoA-I $\mathrm{KO}, 105.3 \pm 19.2 \mathrm{mg} / \mathrm{dl}(n=3)$; probucol-fed wild-type, $33.6 \pm 3.4$ $\mathrm{mg} / \mathrm{dl}(n=9)$; probucol-fed SR-BI KO, $107.8 \pm 6.3 \mathrm{mg} / \mathrm{dl}(n=14)$. All total cholesterol differences greater than $10 \mathrm{mg} / \mathrm{dl}$ were statistically significant $(P<0.05)$.

mating period. When the females exhibited very little or considerably reduced fertility (i.e., SR-BI KO, double SR-BI/apoA-I KO, and double SR-BI/RAG-2 KO mice), the matings were conducted for 4 months. Otherwise shorter 1- to 2-month mating periods were used when the fertile females produced litters relatively quickly.

Ovary transplantation. Donor SR-BI KO or control wild-type mice (5-6 weeks of age) were sacrificed by cervical dislocation, and the ovaries were removed (24). Hosts (RAG-2 KO mice), 4-6 weeks of age, were anesthetized with $2.5 \%$ Avertin (intraperitoneally), and ovary transfer surgery was performed as described previously (24). In brief, ovaries of the host mice were removed from the surrounding ovarian bursa by cutting the side opposite to the ovarian hilus. Ovaries from the donor mice were then inserted into the ovarian bursa and the bursa closed with Vetbond tissue adhesive (3M Animal Care Products, St. Paul, Minnesota, USA). In sham ovary transplants $(n=5)$, ovaries of SR-BI KO mice were removed and reinserted into the same mouse (either unilaterally when the contralateral oviduct was cut, $n=1$, or bilaterally, $n=4$ ). The transplanted hosts were mated with wild-type males 2-3 weeks after surgery and the offspring genotyped by PCR as described above.

\section{Results}

SR-BI expression in the liver is crucial for normal murine lipoprotein metabolism $(16,18,19)$. It extracts cholesterol (as cholesteryl esters) from the hydrophobic cores of circulating plasma HDLs (and other lipoproteins) by a selective uptake pathway $(18,25,26)$, 
thus controlling the plasma levels of HDL cholesterol and the structure of the HDL particles (e.g., restricting the growth in size of the particles by selectively removing core lipid). In SR-BI KO mice there are abnormally high (approximately twofold) levels of plasma total cholesterol, most of which is found in abnormally large, heterogeneous, apoE-enriched HDL-like particles (19). This can be seen in the lipoprotein cholesterol profiles in Figure 1. In these experiments, murine plasma samples were size fractionated (largest lipoprotein particles elute first: VLDL>IDL $>L D L>H D L)$, and the cholesterol content of each fraction was measured. In wild-type females (Figure 1a, dashed line) most of the cholesterol is in normal-size HDL, while in SR-BI KO females Figure 1a, open circles) much of the cholesterol elutes earlier in abnormally large HDL particles. To determine if the abnormal HDLs in SR-BI KO females play a role in their infertility, we used genetic and pharmacologic methods to modify the structure of HDL and lower the amount of plasma HDL cholesterol in these animals, then subsequently examined their fertility by mating them with wild-type males.

We first crossed the SR-BI KO mice with mice that cannot synthesize apoA-I (apoA-I KO mice $(23,27)$, the major - but not the only - protein component of the HDLs in both wild-type and SR-BI KO mice (19). ApoA-I itself is not required for fertility (27). The lipid and apolipoprotein compositions of HDL in apoA-I KO mice differ from those in wild-type mice (e.g., increased apoE in the HDLs of apoA-I KO mice) (27). As reported previously (27), these mice have a low level of plasma cholesterol, the bulk of which is HDL of somewhat larger size than normal HDL (Figure $1 \mathrm{a}$, open triangles). There was a substantial increase in the amount of large, HDL-like cholesterol in the SR-BI/apoA-I double-KO mice (Figure 1a, filled triangles) relative to that in the apoA-I KO mice. This suggests that SR-BI participates directly in the clearance of cholesterol from apoA-I-deficient HDL. It also suggests that, even though there is only a relatively small lipoprotein cholesterol pool in apoA-I KO mice (27), there is nevertheless a substantial, SRBI-dependent flux of cholesterol through this pool. Consistent with this possibility are earlier studies that showed that (a) other apolipoproteins found in HDL (e.g., apoA-II) can serve as ligands for SR-BI (28); and (b) adenovirus-mediated overexpression of SR-BI in apoA-I KO mice results in the loss of virtually all of the apoA-I-deficient HDL cholesterol (K. Kozarsky and $\mathrm{M}$. Donahee, personal communication). There was a reduction in the amount of HDL-like cholesterol in the SR-BI/apoA-I double -KO relative to SR-BI $\mathrm{KO}$ (Figure 1a, open circles) mice, with the greatest reduction seen in the smallest, normal-sized HDL particles (fractions 29-38). Therefore, in the context of a SR-BI KO background, the loss of apoA-I resulted in significant changes in the structure of the HDL (e.g., no apoA-I, altered size distribution) and the amount of HDL cholesterol.
Virgin SR-BI/apoA-I double-KO females (approximately 6-8 weeks of age) were mated with wild-type males for 4 months, and pregnancies and litter sizes counted. During the 4-month mating period, $41 \%$ ( 7 of 17) became pregnant and carried their litters to term (Figure 2, dark gray bars). Observed litter size varied from one to four (average 2.2), and pups from the litters of three or more were nursed properly (smaller litters were cannibalized), developed normally, and had the expected genotypes. The time of mating for the first litter to be born varied from 21 days to 3.5 months (average $56 \pm 12$ days). These results establish that SR-BI is not absolutely required either in the ovaries or extraovarian tissues for successful pregnancies, provided that the extraovarian environment is appropriate (e.g., apoA-I-deficient).

The intrinsic ability of SR-BI-deficient ovaries to produce significant levels of fertilizable oocytes was confirmed in bilateral ovary transplantation experiments in which SR-BI-negative ovaries from SR-BI KO mice were transplanted into ovariectomized SR-BI-positive hosts $(n=7)$. Immunocompromised RAG-2 KO mice (29) were used as hosts to minimize host-versus-graft rejection. Lipoprotein profiles of RAG-2 KO mice, analyzed by FPLC, were virtually identical to those of wildtype mice (data not shown). After mating with wildtype males, six out of seven RAG-2 KO females with SR-BI-negative ovaries (85.7\%) became pregnant and carried the pregnancies to term. The litter sizes varied from one to eight, and all offspring were heterozygous for the SR-BI mutation, confirming that the oocytes originated from the transplanted ovary. Some of the mice became pregnant immediately after being mated to the males only $2-3$ weeks after the transplantation

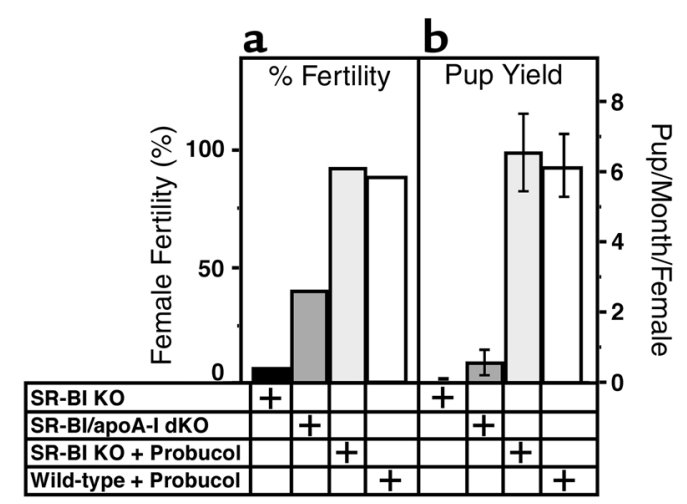

\section{Figure 2}

Effects of genetic disruption of the apoA-I gene or probucol treatment on the fertility of female SR-BI KO mice. Wild-type males were mated with female SR-BI KO ( $n=13$, black bars, average litter size $=1,2$ - to 6-month mating), SR-BI/apoA-I KO ( $n=17$, dark gray bars, average litter size $=2.2$, 4-month mating), probucol-fed SR-BI KO $(n=14$, light gray bars, average litter size $=5.7,1$ - to 2-month mating), and probucol-fed wild-type ( $n=9$, white bars, average litter size $=5.3$, 1- to 2-month mating) mice. (a) Fertility expressed as percentage of females producing litters. (b) Average number of pups delivered per month per female mated (error bars represent SEM). 
surgery. Thus, the SR-BI-negative ovaries very rapidly developed the capacity to produce functional oocytes in an SR-BI-positive environment. No pregnancies were observed during the 4-month mating period in control (sham) surgeries $(n=5)$, thus the surgical manipulation was unlikely to have been responsible for the successful pregnancies. The possibility that the reduced immunological competence of the RAG-2 KO host mice (29) might have potentially rescued the fertility of the SR-BI-negative ovaries was examined by generating SR-BI/RAG-2 double-KO females and mating them with wild-type males. None of the SR-BI/ RAG-2 double-KO mice (plasma lipoprotein profile, analyzed by FPLC, was similar to that of the SR-BI KO mice (data not shown)) that were mated ( $n=6$ for a 4-month period) had litters suggesting that reduced immunological competence was unlikely to have enhanced the fertility of SR-BI-negative ovaries transplanted into the RAG-2 KO mice. Taken together, these results confirm that ovarian expression of SR-BI is not required during embryonic development (when primordial follicles are generated) or for adult maturation and ovarian function (conversion of primordial follicles to antral follicles and ovulation) for production of functional oocytes in an appropriate extraovarian environment (e.g., SR-BI-positive or apoA-I-negative).

It seems likely that apoA-I-dependent changes in the structure and/or composition of the lipoproteins in the SR-BI/apoA-I double-KO females were primarily responsible for the partial restoration of fertility. Similarly, it is tempting to speculate that either the presence of normal lipoproteins or absence of abnormal lipoproteins in the plasma of RAG-2 KO hosts was the major factor enabling the transplanted SR-BI KO ovaries to produce fertilizable oocytes in this otherwise SR-BI-positive environment. Because these animals have different mixed genetic backgrounds, it is possible that some unknown modifier alleles may have influenced fertility in these experiments. To test this independently, we examined the effects on fertility of modifying the abnormal HDL-cholesterol in SR-BI KO mice with the powerful HDL cholesterol-lowering drug probucol (30). As described previously (19), we found that the baseline plasma total cholesterol levels of the SR-BI KO mice were two times higher than those of the wild-type mice (SR-BI KO mice, $n=13,211.5 \pm 6.2$ $\mathrm{mg} / \mathrm{dl}$; wild-type mice, $n=7,103.4 \pm 3.8 \mathrm{mg} / \mathrm{dl}$ ). Probucol treatment reduced plasma cholesterol levels by approximately $67 \%$ in wild-type (Figure $1 \mathrm{~b}$ ) and approximately $49 \%$ in SR-BI KO (Figure 1c) mice without substantially altering the apparent sizes of the HDLs. Thus, the as yet undefined mechanism by which probucol lowers murine plasma cholesterol (and possibly modifies the structure of lipoproteins) $(31,32)$ does not require the expression of SR-BI. Remarkably, probucol-treatment, which began 1-3.5 weeks before mating and continued throughout the experiment, completely restored fertility to the SR-BI KO mice, measured either as percentage of experimental females producing litters (93\%, Figure 2a, light gray bar), or as average number of pups per month delivered per female mated (Figure 2b, light gray bar). Fertility was relatively rapidly restored by drug treatment, as seen by the brief average time from mating to delivery -28.4 days (approximately 19 days of which are accounted for by the typical time for murine gestation). Probucol treatment was significantly more effective in restoring fertility than genetically ablating apoA-I expression (Figure 2, compare light and dark gray bars). These data show that the infertility of SR-BI KO mice is not due to irreversible defects in embryonic or postnatal ovarian development. In addition to its lipid-lowering effects, probucol exhibits antioxidant and possibly other activities $(33,34)$ that might have influenced fertility. However, when the probucol, SR-BI/apoA-I double-KO mice and ovary transfer experiments are considered together, it seems likely that abnormal lipoprotein metabolism is responsible for the infertility of SR-BI KO females and that appropriate modification of the structure/composition/abundance of the lipoproteins can restore fertility in the absence of ovarian and extraovarian SR-BI expression.

\section{Discussion}

SR-BI KO females have abnormal HDLs, ovulate dysfunctional oocytes and are infertile. We used surgical, genetic and pharmacologic methods to show that the fertility of SR-BI KO females (or their transplanted oocytes) can be restored in the absence of ovarian and/or extraovarian SR-BI expression by manipulations that modify the structure, composition and/or abundance of their abnormal plasma lipoproteins. These manipulations included inactivation of the apolipoprotein A-I gene and administration of the cholesterol-lowering drug probucol. Thus, it seems likely that abnormal lipoprotein metabolism is responsible for the reversible infertility of SR-BI KO females.

Many strains of mutant or transgenic mice with alterations in other genes affecting adult lipoprotein metabolism (e.g., LDLR, apoE, LCAT, VLDLR, HL, PLTP, CETP, ABCA1) (35-44) exhibit abnormal increases or decreases in plasma cholesterol comparable or greater in magnitude than that seen in SR-BI KO mice. However, the abnormal lipoprotein cholesterol profile of SR-BI KO mice (Figure 1) appears to be unique. With one exception, these other mice have not been reported to exhibit female infertility. Thus, altered plasma cholesterol per se is not sufficient to cause infertility. The exception is impaired female fertility (placental malformation) of homozygous ATP-binding cassette transporter A1 (ABCA1) KO females (45). These mice have almost no spherical plasma HDL particles, apparently because of defective cholesterol efflux from cells to nascent HDL particles $(43,44)$. While the relationship between the female infertility in SR-BI KO and ABCA1 KO mice remains to be defined, it appears that only very specific abnormalities in lipoprotein structure/composition cause murine female infertility. 
Interestingly, in the "restricted ovulator" (R/O) strain of chickens, an inactivating mutation in the oocyte vitellogenesis receptor results in hyperlipidemia, oocyte growth defects, and female sterility (46). This VLDL receptor-like protein binds both major yolk lipoproteins, VLDL and vitellogenin, and provides a variety of nutrients for embryogenesis (reviewed in refs. 47, 48). Because of the substantial differences in the development, structure, and fate of avian and murine oocytes, the mechanisms underlying the female infertility of the $\mathrm{R} / \mathrm{O}$ chicken and the SR-BI KO mouse may not necessarily be similar.

There are several mechanisms by which lipoproteins could influence fertility in mice. First, it is possible that lipoproteins, especially HDL, normally serve as carriers of important permissive fertility factors in female mice. For example, a candidate component of HDL that might be critical is vitamin $\mathrm{E}$, which is transported in lipoproteins, can be delivered to cells via SR$\mathrm{BI}$ and is required for murine female fertility (49-53). The abnormal plasma lipoproteins in SR-BI KO mice may be unable to provide required fertility factors for normal oocyte production in mice that have no ovarian SR-BI activity. In the case of the SR-BI-negative ovaries transplanted into otherwise SR-BI-positive RAG-2 host animals, the absence of abnormal lipoproteins, presence of normal lipoproteins, or both appears likely to be the cause of the restored fertility. Alternatively SR-BI KO mice may be infertile because their abnormal lipoproteins inhibit critical ovarian functions. Reduction in the amounts of such "toxic" lipoproteins by either the removal of apoA-I by mutation or probucol treatment might restore fertility. In either case, it appears that the liver can influence ovarian function, at least in the case of SR-BI-negative ovaries, because of its importance in controlling lipoprotein metabolism. This raises the possibility that, in addition to the well-studied hypothalamichypophyseal-ovarian, hypothalamic-adrenal-ovarian, and ovarian-uterine axes, an hepatic-ovarian axis may play a role in the ovarian/extraovarian interactions required for fertility.

It is estimated that for $10-20 \%$ of women with infertility problems, the underlying causes are unknown $(2,54)$. We are not aware of any reports exploring the potential etiology of abnormal lipoprotein structure or metabolism in these patients, although a few studies have examined the potential role of lipoproteins in female infertility associated with endocrine disturbances such as polycystic ovarian syndrome $(55,56)$. Because HDL is the only lipoprotein present in substantial amounts in the follicular fluid surrounding the developing oocyte in humans $(8,9)$, it is possible that changes in HDL (structure/composition/abundance) and/or SR-BI structure and function in humans may disturb oocyte maturation or function, and thus contribute to infertility. The analysis of SR-BI KO mice suggests that additional investigation of potential relationships between abnormal lipoprotein metabolism and human female infertility of unknown etiology may be informative and may possibly lead to new therapeutic approaches for some infertile women.

\section{Acknowledgments}

We thank S. Ebrahim, M. Penman, B. Trigatti, M. Matzuk, J. Chen, R. Hynes, and H. Lodish for technical assistance and/or helpful discussions; $\mathrm{B}$. Trigatti for generating the SR-BI/apoA-I double-KO mice; and A. Rigotti, K. Kozarsky, and M. Donahee for permission to cite their unpublished data. This work was supported by grants H-L41484, HL-52212, HL-66105, and HL64737 from the National Institutes' of Health Heart Lung and Blood Institute. H. Miettinen was a Postdoctoral Fellow supported by Academy of Finland, Finnish Cultural Foundation, and Yrjo Jahnsson Foundation.

1. te Velde, E.R., and Cohlen, B.J. 1999. The management of infertility. $N$. Engl. J. Med. 340:224-226.

2. Templeton, A.A., and Penney, G.C. 1982. The incidence, characteristics, and prognosis of patients whose infertility is unexplained. Fertil. Steril. 37:175-182.

3. Azhar, S., et al. 1998. Human granulosa cells use high density lipoprotein cholesterol for steroidogenesis. J. Clin. Endo. Metab. 83:983-991.

4. Lafond, J., et al. 1999. Presence of CLA-1 and HDL binding sites on syncytiotrophoblast brush border and basal plasma membranes of human placenta. Placenta. 20:583-590.

5. Handwerger, S., et al. 1999. Pre-beta-HDL stimulates placental lactogen release from human trophoblast cells. Am. J. Physiol. 276:E384-E389.

6. Farese, R.V.J., and Herz, J. 1998. Cholesterol metabolism and embryogenesis. Trends Genet. 14:115-120.

7. Evans, H.M., and Bishop, K.S. 1922. On the existence of a hitherto unrecognized dietary factor essential for reproduction. Science. 56:650-651.

8. Shalgi, R., Kraicer, P., Rimon, A., Pinto, M., and Soferman, N. 1973. Proteins of human follicular fluid: the blood-follicle barrier. Fertil. Steril. 24:429-434.

9. Perret, B.-P., et al. 1985. Lipoprotein and phospholipid distribution in human follicular fluids. Fertil. Steril. 43:405-409.

10. Le Geoff, D. 1994. Follicular fluid lipoproteins in the mare: evaluation of HDL transfer from plasma to follicular fluid. Biochim. Biophys. Acta. 1210:226-232.

11. Rothblat, G.H., et al. 1999. Cell cholesterol efflux: integration of old and new observations provides new insights. J. Lipid Res. 40:781-796.

12. Schmitz, G., Kaminski, W.E., and Orso, E. 2000. ABC transporters in cellular lipid trafficking. Curr. Opin. Lipidol. 11:493-501.

13. Bodzioch, M., et al. 1999. The gene encoding ATP-binding cassette transporter 1 is mutated in Tangier disease. Nat. Genet. 22:347-351.

14. Brooks-Wilson, A., et al. 1999. Mutations in ABC1 in Tangier disease and familial high-density lipoprotein deficiency. Nat. Genet. 22:336-345.

15. Rust, S., et al. 1999. Tangier disease is caused by mutations in the gene encoding ATP-binding cassette transporter 1. Nat. Genet. 22:352-355.

16. Kozarsky, K.F., et al. 1997. Overexpression of the HDL receptor SR-BI alters plasma HDL and bile cholesterol levels. Nature. 387:414-417.

17. Ji, Y., et al. 1997. Scavenger receptor BI promotes high density lipoprotein-mediated cellular cholesterol efflux. J. Biol. Chem. 272:20982-20985.

18. Krieger, M. 1999. Charting the fate of the "good cholesterol": identification and characterization of the HDL receptor SR-BI. Ann. Rev. Biochem. 68:523-588.

19. Rigotti, A., et al. 1997. A targeted mutation in the murine gene encoding the high density lipoprotein (HDL) receptor scavenger receptor class B type I reveals its key role in HDL metabolism. Proc. Natl. Acad. Sci. USA. 94:12610-12615.

20. Trigatti, B., et al. 1999. Influence of the high density lipoprotein receptor SR-BI on reproductive and cardiovascular pathophysiology. Proc. Natl. Acad. Sci. USA. 96:9322-9327.

21. Hazopoulos, A., Rigotti, A., Rosenberg, R., and Krieger, M. 1998. Temporal and spatial pattern of expression of the HDL receptor SR-BI during murine embryogenesis. J. Lipid Res. 39:495-506.

22. Landschulz, K., Pathak, R., Rigotti, A., Krieger, M., and Hobbs, H. 1996. Regulation of scavenger receptor, class B, type I, a high density lipoprotein receptor, in liver and steroidogenic tissues of the rat. J. Clin. Invest. 98:984-995.

23. Williamson, R., Lee, D., Hagaman, J., and Maeda, N. 1992. Marked reduction of high density lipoprotein cholesterol in mice genetically modified to lack apolipoprotein A-I. Proc. Natl. Acad. Sci. USA. 89:7134-7138. 
24. Hogan, B., Beddington, R., Costantini, F., and Lacy, E. 1994. In Manipulating the mouse embryo. Cold Spring Harbor Press. Plainview, New York, USA. 130-188.

25. Glass, C., Pittman, R.C., Weinstein, D.B., and Steinberg, D. 1983. Dissociation of tissue uptake of cholesterol ester from that of apoprotein A-I of rat plasma high density lipoprotein: selective delivery of cholesterol ester to liver, adrenal, and gonad. Proc. Natl. Acad. Sci. USA. 80:5435-5439.

26. Acton, S., et al. 1996. Identification of scavenger receptor SR-BI as a high density lipoprotein receptor. Science. 271:518-520.

27. Li, H., Reddick, R.L., and Maeda, N. 1993. Lack of apo A-I is not associated with increased susceptibility to atherosclerosis in mice. Arterioscler. Thromb. 13:1814-1821.

28. Xu, S., et al. 1997. Apolipoproteins of HDL can directly mediate binding to the scavenger receptor SR-BI, an HDL receptor that mediates selective lipid uptake. J. Lipid Res. 38:1289-1298.

29. Shinkai, Y., et al. 1992. RAG-2-deficient mice lack mature lymphocytes owing to inability to initiate V(D)J rearrangement. Cell. 68:855-867.

30. Barnhart, J.W., Sefranka, J.A., and McIntosh, D.D. 1970. Hypocholesterolemic effect of 4,4'-(isopropylidenedithio) bis(2,6-di-t-bytylphenol) (probucol). Am. J. Clin. Nutr. 23:1229-1233.

31. Rinninger, F., Wang, N., Ramakrishnan, R., Jiang, X.C., and Tall, A.R. 1999. Probucol enhances selective uptake of HDL-associated cholestery esters in vitro by a scavenger receptor B-I-dependent mechanism. Arterioscler. Thromb. Vasc. Biol. 19:1325-1332.

32. Tsujita, M., Tomimoto, S., Okumura-Noji, K., Okazaki, M., and Yokoyama, S. 2000. Apolipoprotein-mediated cellular cholesterol/phospholipid efflux and plasma high density lipoprotein level in mice. Biochim. Biophys. Acta. 1485:199-213.

33. Honda, K., et al. 2000. Induction of estradiol synthesis by probucol in the adenocarcinoma cells of an ovarian clear cell tumor. Anticancer Res. 20:4397-4401.

34. Zimetbaum, P., Eder, H., and Frishman, W.J. 1990. Probucol: pharmacology and clinical application. Clin. Pharmacol. 30:3-9.

35. Ishibashi, S., et al. 1993. Hypercholesterolemia in low density lipoprotein receptor knockout mice and its reversal by adenovirus-mediated gene delivery. J. Clin. Invest. 92:883-893.

36. Plump, A.S., et al. 1992. Severe hypercholesterolemia and atherosclerosis in apolipoprotein E-deficient mice created by homologous recombination in ES Cells. Cell. 71:343-353.

37. Zhang, S.H., Reddick, R.L., Piedrahita, J.A., and Maeda, N. 1992. Spontaneous hypercholesterolemia and arterial lesions in mice lacking apolipoprotein E. Science. 258:468-471.

38. Vaisman, B.L., et al. 1995. Overexpression of human lecithin cholesterol acyltransferase leads to hyperalphalipoproteinemia in transgenic mice. J. Biol. Chem. 270:12269-12275.

39. Frykman, P.K., Brown, M.S., Yamamoto, T., Goldstein, J.L., and Herz, J. 1995. Normal plasma lipoproteins and fertility in gene-targeted mice homozygous for a disruption in the gene encoding very low density lipoprotein receptor. Proc. Natl. Acad. Sci. USA. 92:8453-8457.

40. Homanics, G.E., et al. 1995. Mild dyslipidemia in mice following targeted inactivation of the hepatic lipase gene. J. Biol. Chem. 270:2974-2980.

41. Jiang, X.C., et al. 1999. Targeted mutation of plasma phospholipid transfer protein gene markedly reduces high-density lipoprotein levels. J. Clin Invest. 103:907-914.

42. Agellon, L.B., et al. 1991. Reduced high density lipoprotein cholesterol in human cholesteryl ester transfer protein transgenic mice. J. Biol. Chem. 266:10796-10801.

43. Orso, E., et al. 2000. Transport of lipids from golgi to plasma membrane is defective in tangier disease patients and Abc1-deficient mice. Nat. Genet. 24:192-196.

44. McNeish, J., et al. 2000. High density lipoprotein deficiency and foam cell accumulation in mice with targeted disruption of ATP-binding cassette transporter-1. Proc. Natl. Acad. Sci. USA. 97:4245-4250.

45. Christiansen-Weber, T.A., et al. 2000. Functional loss of ABCA1 in mice causes severe placental malformation, aberrant lipid distribution, and kidney glomerulonephritis as well as high-density lipoprotein cholesterol deficiency. Am. J. Pathol. 157:1017-1029.

46. Bujo, H., et al. 1995. Mutant oocytic low density lipoprotein receptor gene family member causes atherosclerosis and female sterility. Proc. Natl. Acad. Sci. USA. 92:9905-9909.

47. Bujo, H., Hermann, M., Lindstedt, K.A., Nimpf, J., and Schneider, W.J. 1997. Low density lipoprotein receptor gene family members mediate yolk deposition. J. Nutr. 127(Suppl. 5):801S-804S

48. Schneider, W.J., Osanger, A., Waclawek, M., and Nimpf, J. 1998. Oocyte growth in the chicken: receptors and more. Biol. Chem. 379:965-971.

49. Kolleck, I., et al. 1999. HDL is the major source of vitamin E for type II pneumocytes. Free Radic. Biol. Med. 27:882-890.

50. Jishage Ki, K., et al. 2000. Alpha-tocopherol transfer protein is important for the normal development of placental labyrinthine trophoblasts in mice. J. Biol. Chem. 276:1669-1672.

51. Terasawa, Y., et al. 2000. Increased atherosclerosis in hyperlipidemic mice deficient in $\alpha$-tocopherol transfer protein and vitamin E. Proc. Natl. Acad. Sci. USA. 97:13830-13834.

52. Goti, D., et al. 2001. Scavenger receptor class B, type I is expressed in porcine brain capillary endothelial cells and contributes to selective uptake of HDL-associated vitamin E. J. Neurochem. 76:498-508.

53. Mardones, P., et al. 2001. Role of the scavenger receptor class B type I (SR-BI) in a-tocopherol metabolism in mice. J. Nutr. In press.

54. Templeton, A. 2000. Infertility and the establishment of pregnancyoverview. British Med. Bull. 56:577-587.

55. Hansen, K.K., Knopp, R.H., and Soules, M.R. 1991. Lipoprotein-cholesterol levels in infertile women with luteal phase deficiency. Fertil. Steril. 55:916-921.

56. Rajkhowa, M., et al. 1997. Altered composition of high density lipoproteins in women with the polycystic ovary syndrome. J. Clin. Endocrinol. Metab. 82:3389-3394. 